\title{
Biofunctionalized all-polymer photonic lab on a chip with integrated solid-state light emitter
}

\author{
Andreu Llobera ${ }^{1,2}$, Joan Juvert ${ }^{1}$, Alfredo González-Fernández ${ }^{1}$, Bergoi Ibarlucea ${ }^{1}$, Ester Carregal-Romero ${ }^{1}$, \\ Stephanus Büttgenbach ${ }^{2}$ and César Fernández-Sánchez ${ }^{1}$
}

\begin{abstract}
A photonic lab on a chip (PhLOC), comprising a solid-state light emitter (SSLE) aligned with a biofunctionalized optofluidic multiple internal reflection (MIR) system, is presented. The SSLE is obtained by filling a microfluidic structure with a phenyltrimethoxysilane (PhTMOS) aqueous sol solution containing a fluorophore organic dye. After curing, the resulting xerogel solid structure retains the emitting properties of the fluorophore, which is evenly distributed in the xerogel matrix. Photostability studies demonstrate that after a total dose (at $\lambda=365 \mathrm{~nm}$ ) greater than $24 \mathrm{~J} \mathrm{~cm}^{-2}$, the xerogel emission decay is only $4.1 \%$. To re-direct the emitted light, the SSLE includes two sets of air mirrors that surround the xerogel. Emission mapping of the SSLE demonstrates that alignment variations of $150 \mu \mathrm{m}$ (between the SSLE and the external pumping light source) provide fluctuations in emitted light smaller than $5 \%$. After this verification, the SSLE is monolithically implemented with a MIR, forming the PhLOC. Its performance is assessed by measuring quinolone yellow, obtaining a limit of detection (LOD) of $(0.60 \pm 0.01) \mu \mathrm{M}$. Finally, the MIR is selectively biofunctionalized with horseradish peroxidase (HRP) for the detection of hydrogen peroxide $\left(\mathrm{H}_{2} \mathrm{O}_{2}\right)$ target analyte, obtaining a LOD of $(0.7 \pm 0.1) \mu M$ for $\mathrm{H}_{2} \mathrm{O}_{2}$, confirming, for the first time, that solid-state xerogel-based emitters can be massively implemented in biofunctionalized PhLOCs.

Light: Science \& Applications (2015) 4, e271; doi:10.1038/Isa.2015.44; published online 10 April 2015
\end{abstract}

Keywords: medical devices; photonic lab on a chip; polymer technology; solid-state light emitters; sol-gel

\section{INTRODUCTION}

Since the introduction of the $\mu$ TAS concept by Manz and co-workers in $1990,{ }^{1}$ there has been a steady development of this research line, from the definition of a wide range of architectures and systems ${ }^{2}$ to the application of new materials $s^{3,4}$ and the implementation of versatile production technologies. ${ }^{5}$ The reduction of size resulted not only in a decrease in the analysis time and reagent consumption, but also in an enhancement of the sensitivity and possibility of parallelizing the analysis without dramatically increasing both the complexity and overall size of the system. Then, the $\mu$ TAS concept was further expanded to the lab on a chip (LOC), ${ }^{6}$ where all (or most of) the required steps in an analytical protocol can be pursued. These systems have increasingly been applied in many different fields such as drug discovery and development, ${ }^{7}$ genomics, ${ }^{8}$ clinical diagnosis ${ }^{9}$ and cellomics. ${ }^{10}$ In most of these examples, an inexpensive, disposable system with superior performance in terms of sensitivity to a specific analyte is required. Reduction of fabrication costs is generally associated with the use of polymeric materials and simple fabrication techniques, among which poly(dimethylsiloxane) (PDMS) and soft lithography ${ }^{11}$ are excellent examples, respectively. PDMS has widely been applied in the development of LOCs due to its fabrication simplicity. This elastomeric mater- ial can be easily patterned by soft lithography. Furthermore, PDMS is mechanically robust, exhibits a low Young's modulus (300-800 kPa), exhibits a low refractive index $(n=1.41)$ and is transparent in the UVNIR range. However, PDMS is also associated with some important drawbacks when being applied for biochemical analysis that are related to its hydrophobic character, which makes the material very prone to serious nonspecific absorption processes of different organic molecules and biomolecules. Here, several surface modification processes have been reported, such as oxygen plasma ${ }^{12}$ or UV/ozone treatments, ${ }^{13}$ which produce silanol groups $(\mathrm{Si}-\mathrm{OH})$ on the material surface and allow tuning of its hydrophilic/hydrophobic balance. These groups are dynamic, and the surface recovers its hydrophobicity with time. Therefore, subsequent chemical processes must be readily applied to introduce other functional groups to which different (bio)chemical compounds can be firmly attached. ${ }^{12}$ In a different approach, hydroxyl $(-\mathrm{OH})$ groups are introduced on the PDMS surface by physisorption of polyvinyl alcohol (PVA) polymer ${ }^{14}$ and further silanization ${ }^{15}$ of the resulting surface. This process allows the introduction of functional chemical groups that enables the eventual covalent attachment of biomolecules, ${ }^{16-18}$ with the final aim of implementing a microbioreactor (understood as a biofunctionalized microfluidic element) in the LOC

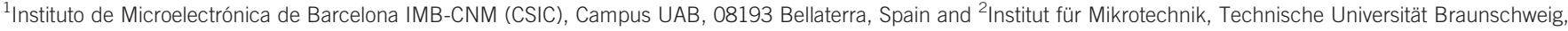
Braunschweig 38106, Germany

Correspondence: A Llobera, Instituto de Microelectrónica de Barcelona IMB-CNM (CSIC), Campus UAB, 08193 Bellaterra, Spain

E-mail: andreu.Ilobera@imb-cnm.csic.es

C Fernández-Sánchez, Instituto de Microelectrónica de Barcelona IMB-CNM (CSIC), Campus UAB, 08193 Bellaterra, Spain

E-mail: cesar.fernandez@imb-cnm.csic.es

Received 23 October 2014; revised 16 December 2014; accepted 10 January 2015; accepted article preview online 20 January 2015 
that provides the desired selectivity for a specific analyte while minimizing interference due to non-specific adsorption of other species.

Equally important is defining the most appropriate transducer to transform this biorecognition event into a quantifiable signal. Here, different transduction mechanisms, such as electrochemical, ${ }^{19}$ magnetic ${ }^{20}$ and optic/photonic, ${ }^{21}$ have been integrated into LOCs. Among these options, optical transducers have been demonstrated to be one of the most sensitive analytical and selective detection methods in cell biology. ${ }^{22}$ Transference of this know-how from the macro- to the microscale has already been demonstrated with the so-called photonic lab on a chip (PhLOC). ${ }^{23}$ Micro-optical elements, such as microlenses and mirrors, ${ }^{24}$ can be implemented in the vicinity of a microfluidic network to provide the entire system with the required optical readout. Here, an open issue is to provide a mechanically robust, optically efficient and technologically simple method that allows light incoupling. Alignment between the light source and optical system is by far the most critical aspect in the PhLOC because small variations (less than $5 \%$ ) of their relative positions cause unacceptable light coupling variations. This key aspect likely hampers the massive implementation of the PhLOC concept and restricts its application to research labs, where the alignment can accurately be controlled with the help of micropositioners. One first step toward tackling this issue is to define self-alignment microchannels for the clamping and positioning of fiber optics. ${ }^{25}$ Although effective, this approach requires trained users and special tools to define high-quality fiber optic facets, not to mention their inherent fragility. The optimal configuration would be to have a disposable light source directly implemented (and hence aligned) in the PhLOC. Here, two different strategies could be pursued, namely, either heterogeneous or monolithic integration. In the first case, the final system is obtained by the combination of several elements defined in different substrates. An example could be the integration of (organic) light-emitting diodes, (O)LEDs, ${ }^{26}$ or laser dyes. ${ }^{27}$ Despite their good performance, such devices are normally multilayered, requiring several clean-room fabrication steps. In addition, the alignment issue is not solved, resulting in limited fabrication yield and a dramatic increase of the production costs. Conversely, monolithic integration provides inherent aligned structures because of the accurate positioning of the different elements at the photomask level. Despite being a more elegant technology compared with heterogeneous integration, the challenges associated with this approach are much greater. Silicon substrates have proven to be unsuitable for defining optical elements working in the visible range: the high absorbance in the UV-Vis range limits their application to photonics working in the near infrared (above $1100 \mathrm{~nm}$ ), whereas the indirect bandgap hampers their use for implementing light sources. Hybrid monolithic integration can be an elegant alternative, as demonstrated by Balslev and co-workers, ${ }^{21}$ who defined a complete PhLOC, whereby a light source was integrated using a liquid dye laser and waveguides and fluidic microchannels were defined with a photostructurable polymer. Nevertheless, liquid dyes are extremely toxic, and thus, their combination with life science applications is not trivial. This issue is especially evident when using PDMS. The integration of liquid dyes with PDMS proves to be difficult, as this material suffers from severe swelling against most organic solvents and is permeable to both gases and small molecules (such as dyes). Therefore, alternatives to liquidbased light sources must be sought.

Solid-state light emitters (SSLEs), which are mechanically and chemically stable, could be an alternative to liquid dyes. Here, different materials, such as hybrid organic-inorganic polymers, have proven to be good candidates. ${ }^{28}$ Among the candidates, those fabricated using sol-gel technology consist of solid backbone networks into which active chemical species can be easily incorporated ${ }^{29}$ while preserving their (bio)functionality. The optical properties of such materials (refractive index and spectral response) can be tailored as a function of the nature of the different monomers and their ratio. ${ }^{30}$ Moreover, the material processing can be tuned to be compatible with microfabrication techniques, and micro- and nanostructures can be defined with smooth and crack-free surfaces. This last point becomes essential for the fabrication of high-quality photonic elements and systems. ${ }^{31}$ Not surprisingly, sol-gel technology has already been used to define waveguides $^{32}$ and microlenses; ${ }^{33}$ however, to the best of our knowledge, this approach has not been used to implement sol-gel-based light emitters in LOC devices.

In this work, we present the hybrid monolithic integration of a xerogel-based SSLE and an optofluidic multiple internal reflection (MIR) to produce a PhLOC. Although having an advanced configuration, the entire PhLOC only requires a single mask and single substrate technology. To verify the proposed approach, Atto 390 fluorophore has been used to define the SSLE, whereas either non-functionalized or enzymatically functionalized MIR has been implemented. This is the first time, to the best of our knowledge, that feasible integration of this type of light source and its successful performance for bioanalytical applications has been presented using an all-polymer technology.

\section{MATERIALS AND METHODS}

\section{Chemicals}

A PDMS Sylgard 184 elastomer kit was purchased from Dow Corning (Midland, MI, USA) and used according to the datasheet. SU-8 2050 and PGMEA (propylene glycol methyl ether acetate) were acquired from Microresist (Berlin, Germany). The 99\% PVA, 99\% triethylamine, Tween 20, phenyltrimethoxysilane (PhTMOS), dimethyl sulfoxide, horseradish peroxidase (HRP type VI), 2,2'azino-bis(3ethylbenzthiazoline-6-sulfonic acid) (ABTS), and quinoline yellow dye were purchased from Sigma-Aldrich Co. (St Louis, MO, USA). The 90\% 11-triethoxysilyl undecanal was purchased from ABCR GmbH \& Co. KG (Karlsruhe, Germany). The Atto 390 fluorophore $\left(\lambda_{\mathrm{abs}}=390 \mathrm{~nm} ; \lambda_{\mathrm{fl}}=479 \mathrm{~nm}\right.$, in water) was purchased from Atto-Tec $\mathrm{GmbH}$ (Siegen, Germany).

\section{Design-I: SSLE}

Figure 1a shows the SSLE design, which consists of a pear-shaped microfluidic element (height: $230 \mu \mathrm{m}$, total volume: $2.12 \mu \mathrm{L}$ ), two diamond-shaped internal reservoirs and inlet/outlet ports. A set of two air mirrors was also defined to enhance the efficiency of the coupling to an arbitrary optofluidic system (also included in the PhLOC, as discussed below). The SSLE concept is as follows. Assuming that the microfluidic element is filled with a fluorophore (either in liquid or solid phase), when externally pumped with the appropriate wavelength, light will be emitted in $4 \pi$ steradians ( $\mathrm{Sr}$ ). This behavior is suboptimal if the idea is to couple as much emitted light as possible in an optofluidic system close to the SSLE. Thus, the role of the two air mirrors is twofold. First, the mirrors allow multiple interactions between the excitation wavelength and the SSLE, thus enhancing the photonic conversion. Second, the mirrors redirect the emission towards the SSLE tip, allowing a more efficient light coupling to the optofluidic system. The working principle of the air mirrors is illustrated in Figure 1b. Considering the refractive indices of PDMS $(n=1.41)$ and air $(n=1.00)$, if the light reaches the flat air mirror \#1 at an angle higher than the critical angle $\left(\theta_{1}>\theta_{c}=45.17^{\circ}\right)$, the light undergoes total internal reflection and will be redirected back to the SSLE. 

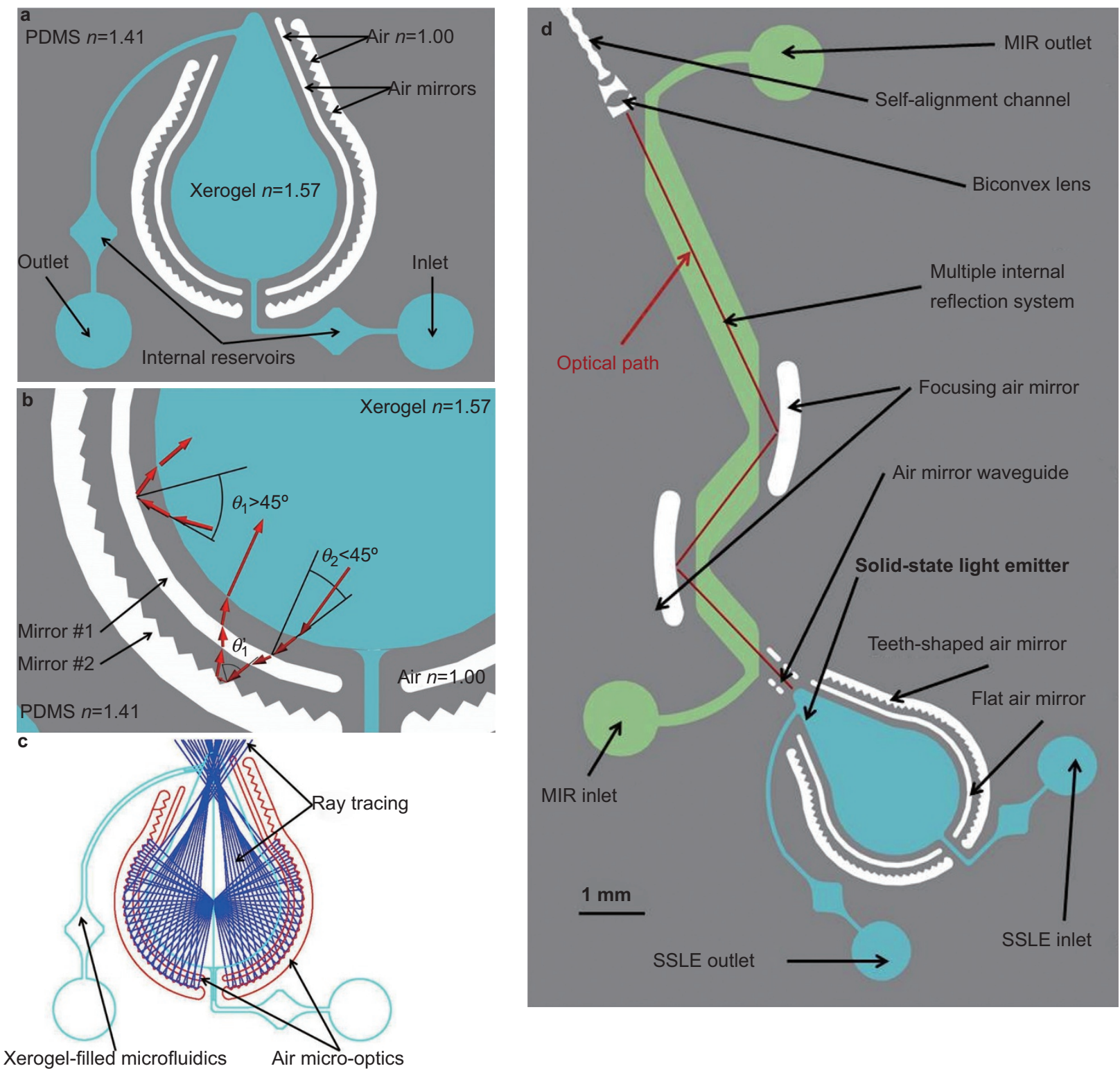

Figure 1 (a) Design of the SSLE using PDMS (gray) as the structural material, fluorophore-doped xerogel (blue), and air (white) for defining the micro-optics elements. (b) Scheme of the working principle of the two air mirrors, considering incidence angles larger $\left(\theta_{1}, \theta_{1}^{\prime}\right)$ and smaller $\left(\theta_{2}\right)$ than the critical angle $\left(\theta_{c}\right)$. (c) Ray tracing of the light emitted from the SSLE and its focusing at the tip. (d) Design of the PhLOC, including the SSLE, the MIR, the interconnecting waveguide, a biconvex microlens and a self-alignment microchannel for inserting the fiber optics readout. The optical path is shown in red. PDMS, poly(dimethylsiloxane); PhLOC, photonic lab on a chip; SSLE, solid-state light emitter.

Smaller angles $\left(\theta_{2}<\theta_{c}\right)$ are transmitted through the first mirror according to the Fresnel laws, reaching the teeth-shaped mirror \#2. The faces of each tooth in mirror \#2 work jointly to reflect angles transmitted through mirror \#1. One of the faces is tilted to ensure total internal reflection at the light incidence point $\left(\theta_{1}^{\prime}>\theta_{c}\right)$, whereas the second face back-reflects the light into the SSLE. In addition, the relative angle of each tooth varies radially along the SSLE to focus the light towards its tip, as observed in the ray-tracing simulation of the teeth-shaped mirrors working as a whole depicted in Figure 1c.

\section{Design-II: PhLOC}

The disposable PDMS-based PhLOC is illustrated in Figure 1d and consists of the previously described SSLE directly aligned to a MIR optofluidic system ${ }^{23}$ interconnected with the help of a waveguide. Both the MIR and waveguide make use of air mirrors whose working principle is identical to that of mirror \#1, the single difference being that the light propagation inside the MIR follows a zigzag path aided by focusing air mirrors (allowing the optical path to be enlarged and thus enhancing its sensitivity without a dramatic increase of the required sample volume). ${ }^{24}$ Flat air mirrors are located at both sides of the bulk PDMS, forming an interconnecting waveguide between the SSLE and MIR. Finally, transmitted light is collected at the end of the MIR with the help of two PDMS microlenses (implemented using only air and PDMS as constituent materials) and fiber optics positioned in a self-alignment microchannel. 


\section{Fabrication}

The PhLOC was fabricated using PDMS and soda-lime glass substrates. The process comprised three main steps, mainly the definition of the master using a photopatternable polymer, replica molding of the master using PDMS, and implementation of the SSLE using a fluorophore-doped organic-inorganic polymer by sol-gel technology. ${ }^{34}$ The first two steps make use of standard techniques, and a detailed description can be found elsewhere. ${ }^{35}$ Briefly, a 230 - $\mu$ m-thick layer of SU-8 2050 was spun over a substrate. After the soft bake, it was exposed to UV light using the appropriate mask. Then, a postexposure bake was performed to crosslink the polymeric structure. Once finished, the wafers were immersed in PGMEA and rinsed vigorously until fully developed. To enhance the mechanical robustness of the masters and to heal possible microcracks generated by internal stresses, a hard bake was performed for $2 \mathrm{~h}$ at $120{ }^{\circ} \mathrm{C}$ in an inert atmosphere. PDMS was prepared by mixing the base elastomer and curing agent in the standard ratio of 1:10 $(v / v)$. Vigorous stirring and applied vacuum assured a bubble-free pre-polymer. After pouring the PDMS onto the master, a second vacuum step was applied to ensure that no air bubbles remained entrapped between the master and PDMS. The PDMS was cured at $80{ }^{\circ} \mathrm{C}$ for $20 \mathrm{~min}$. and then peeled off using tweezers. At this point, all the fluidic ports were opened using a dispenser tip $(\varphi 0.5 \mathrm{~mm})$. Then, the PDMS and a flat pre-cleaned glass substrate were activated with oxygen plasma (PVA TePla AG, Munich, Germany) at $500 \mathrm{~W}$ and $0.8 \mathrm{mbar}$ for $18 \mathrm{~s}$. Afterwards, they were placed in contact using deionized water as a lubricant. By thermal processing at $80{ }^{\circ} \mathrm{C}$ for $30 \mathrm{~min}$, both the PDMS and glass substrate were irreversibly sealed. Once the bonding quality was verified by eye inspection, the SSLE was ready to be filled with the fluorophore-doped pre-polymerization sol solution, which is an adaptation of the micromolding in capillaries soft-lithographic technique. ${ }^{11}$

Among the myriad different fluorophores suitable for this application, Atto 390 was selected because of its high molecular absorption (24.000 $\left.\mathrm{cm}^{-1} \mathrm{M}^{-1}\right)$ and quantum yield $(0.90)$ as well as its large Stokes shift ( $\lambda_{\mathrm{abs}}=390 \mathrm{~nm}, \lambda_{\mathrm{fl}}=479 \mathrm{~nm}$, in water). Atto 390 is slightly hydrophilic and is soluble in polar solvents such as DMF or dimethyl sulfoxide. Solubility was important to ensure compatibility with different chemical environments, which is crucial to obtain a homogeneous dispersion of the fluorophore molecules in the polymeric material. Nevertheless, the technology presented here is not restricted to Atto 390; other fluorophores or dyes with similar properties could also be used.

The fluorophore-doped hybrid organic-inorganic polymer was synthesized using sol-gel technology. ${ }^{34}$ The process involved three main steps: mixing, gelation and drying. During the mixing step, the pre-polymerization sol solution was prepared by vigorously mixing $500 \mu \mathrm{L}$ of PhTMOS monomer with $300 \mu \mathrm{L}$ of a $500 \mu \mathrm{M}$ Atto 390 solution prepared in $\mathrm{H}_{2} \mathrm{O}$ /dimethyl sulfoxide 5:1 $(v / v) \mathrm{pH} 3$ (adjusted with diluted $\mathrm{HCl}$ ). During this step, the monomer was hydrolyzed and started to condense, forming siloxane bonds. An acidic medium was needed to accelerate and favor the hydrolysis of the PhTMOS monomer. At this point, the sol solution was formed. Because the sol is a lowviscosity liquid, filling of the SSLE fluidic structure was achieved by capillary forces, thus avoiding the use of external pumps. Once this microstructure was filled, it was protected from light and left undisturbed to allow the polymer to dry at room temperature for $72 \mathrm{~h}$. During gelation, the viscosity increased sharply, and the solvents in the solution evaporated and diffused through the PDMS stamp. Consequently, the final xerogel had a significantly smaller volume compared with the sol solution. When not properly addressed, this effect led to a partial emptying of the SSLE. In our case, the diamondshaped internal reservoirs assured additional material supply during the gelification step, thus impeding the SSLE emptying. Once the solgel polymeric material dried, a xerogel matrix with entrapped Atto 390 fluorophore was obtained.

\section{Fluorophore-doped polymer characterization}

Fluorophore homogeneous dispersion in the SSLE is of key importance for obtaining a PhLOC with identical performance. In this context, confocal microscopy analysis was performed using a Leica TCS SP2 confocal microscope (Leica Geosystems AG, St Gallen, Switzerland) including an HC PL APO CS $10.0 \times$ UV objective with a 0.40 numerical aperture and a UV $405 \mathrm{~nm}$ diode laser as a light source. The scanned area was $600 \mu \mathrm{m} \times 600 \mu \mathrm{m}$ (width $\times$ length), and images were captured every $5 \mu \mathrm{m}$ in depth with a resolution of 8 bits. Up to 50 pictures were taken to cover all the SSLE thickness.

\section{Photostability}

The SSLE photostability was analyzed by irradiation of the structure with UV light at controlled irradiation doses while acquiring emission spectra. The recorded spectra were then compared with the reference spectrum, and the percentages of the initial emission intensity at the maximum were calculated. The set-up for this study included a mask aligner as a light source (a MA1006 contact mask aligner equipped with a 350-W mercury lamp, $2-\mu \mathrm{m}$ resolution and a split field microscope with $5 \times$ and $10 \times$ objectives, exposure dose of $9 \mathrm{~mJ} \mathrm{~cm}{ }^{-2}$ at $\lambda=365 \mathrm{~nm}$ (i-line); Suss MicroTec, Munich, Germany) and a 230$\mu \mathrm{m}$-diameter multimode optical fiber (Thorlabs Inc., Dachau, Germany) for collecting the light, coupled to a microspectrometer (QE 65000-FL; Ocean Optics, Dunedin, FL, USA). Each spectrum was acquired with an average of 10 scans, and the integration time was fixed at $100 \mathrm{~ms}$. Because the power density is constant in the mask aligner, different irradiation doses were achieved by irradiating the sample at 21 different time intervals between 10 and $2700 \mathrm{~s}$ (equivalently, to optical doses between $90 \mathrm{~mJ} \mathrm{~cm}^{-2}$ and $24.3 \mathrm{~J} \mathrm{~cm}^{-2}$ ).

\section{Mapping emission}

The optimal excitation point and alignment tolerances at which the emitted light intensity has its maximum value at the SSLE tip are key in validating the emitter configuration proposed in this paper. To this effect, the SSLE was also fabricated in a separate substrate, consisting of only the pear-shaped structure, the two air mirrors, the interconnection waveguide, the biconvex microlens and the output self-alignment system (a schema of the structure is provided in Supplementary Fig. S1). Once the doped xerogel was obtained inside the SSLE, a blue laser ( $\lambda=405 \mathrm{~nm}$, Laser module NANO 250-532-100; Linos Photonics, Eschbach, Germany) was coupled to a multimode $230 \mu \mathrm{m}$ optical fiber, which was positioned on a motorized XY stage (VT-80, PiMicos, Eschbach, Germany, Thorlabs, Dachau/Munich, Germany) perpendicular to the plane of the SSLE at a fixed distance of $z=1 \mathrm{~mm}$. The readout comprised an identical optical fiber inserted into the output self-alignment microchannel, coupled to a microspectrometer (QE 65000-FL; Ocean Optics, Dunedin, FL, USA). This configuration allowed geometric localization of the spots in the SSLE and simultaneous acquisition of the spectral response. Each spectrum was acquired with an average of 10 scans, and the integration time was fixed at $250 \mathrm{~ms}$. This process resulted in an efficiency map of the SSLE emission as a function of the excitation position with a resolution of $150 \mu \mathrm{m}$. The study was repeated with four SSLE structures with different Atto concentrations (10, 50, 100 and $500 \mu \mathrm{M})$. 


\section{Biofunctionalization}

The immobilization of HRP as a biorecognition element on the PDMS walls of the MIR structure was performed following the protocol described in Ibarlucea et al. ${ }^{16}$ Briefly, the inner walls of the MIR were modified by direct adsorption of PVA linear polymer. This process introduced hydroxyl groups onto the PDMS surface, which were further silanized using the 11-triethoxysilyl undecanal silane containing an aldehyde end-group. ${ }^{36}$ The covalent interaction between this group and a primary amine moiety of the protein structure enabled the selective robust attachment of the HRP enzyme on the PDMS surface.

\section{Experimental set-up for the $\mathrm{PhLOC}$ and protocol for} absorbance measurements

Light emitted from the blue laser $(\lambda=405 \mathrm{~nm}$, Laser module NANO 250-532-100; Linos Photonics) was coupled with a multimode $230 \mu \mathrm{m}$ optical fiber, which was positioned at the maximum emission region of the SSLE, in accordance with the previously described mapping study. The readout comprised an identical optical fiber, which was inserted into the output self-alignment microchannel of the MIR system and connected to the microspectrometer (QE 65000-FL; Ocean Optics, Dunedin, FL, USA). The acquisition time was fixed at $400 \mathrm{~ms}$, and each recorded spectrum was the average of 10 scans.

The performance of the PhLOC was first tested by sequentially injecting quinoline yellow dye aqueous solutions in a concentration range from 1 to $25 \mu \mathrm{M}$. The readout was recorded immediately after filling the PhLOC, and deionized water was used as a reference.

For final PhLOC validation, biofunctionalized MIRs were tested by sequentially injecting $30 \mu \mathrm{L}$ of $0.1 \mathrm{M}$ acetate buffer $\mathrm{pH} 5.5$ solutions containing $0.5 \mathrm{mM}$ ABTS and increasing concentrations of $\mathrm{H}_{2} \mathrm{O}_{2}$ in the range from $1.4 \mu \mathrm{M}$ to $27.5 \mu \mathrm{M}$. The readout signal of the acetate buffer electrolyte solution was used as the reference. The measurements were made under quiescent fluid conditions (absence of flow) in all cases. The generation of the colored product was time dependent because a delay time was required for the catalytic reaction to occur and the resulting enzymatic product to diffuse to the bulk of the solution inside the MIR. A 10-min stabilization time was selected. Reproducibility studies were performed by calibrating three different biofunctionalized PhLOCs on three consecutive days.

The results of both analytical studies described above were analyzed following the protocol presented in Ref. 35. Once the corresponding absorbance $v s$. concentration calibration curves were obtained, the limit of detection (LOD) could be estimated following the $3 \sigma$ IUPAC criteria. $^{37}$

\section{RESULTS AND DISCUSSION}

\section{Fluorophore-doped polymeric SSLE}

The results from the confocal microscopy lambda analysis are presented in Figure 2. Thirteen different emission spectra at different arbitrary points of the microstructure were recorded; the mean values for the maximum fluorescence emission intensity and wavelength were $(219 \pm 6)$ a.u. (3\% coefficient of variation, number of spectra $=13)$ and $(451 \pm 1) \mathrm{nm}$, respectively. These results indicate that the fluorophore was homogeneously dispersed in the polymeric material.

A $3 \mathrm{D}$ reconstruction of the SSLE emitted light intensity was performed, the projection of which is presented in Figure 2b. As observed, the fluorescence emission is constant across the structure, which means the doped xerogel completely filled the SSLE PDMS structure. This effect can again be associated with the homogeneous fluorophore distribution on a xerogel SSLE with a constant thickness.
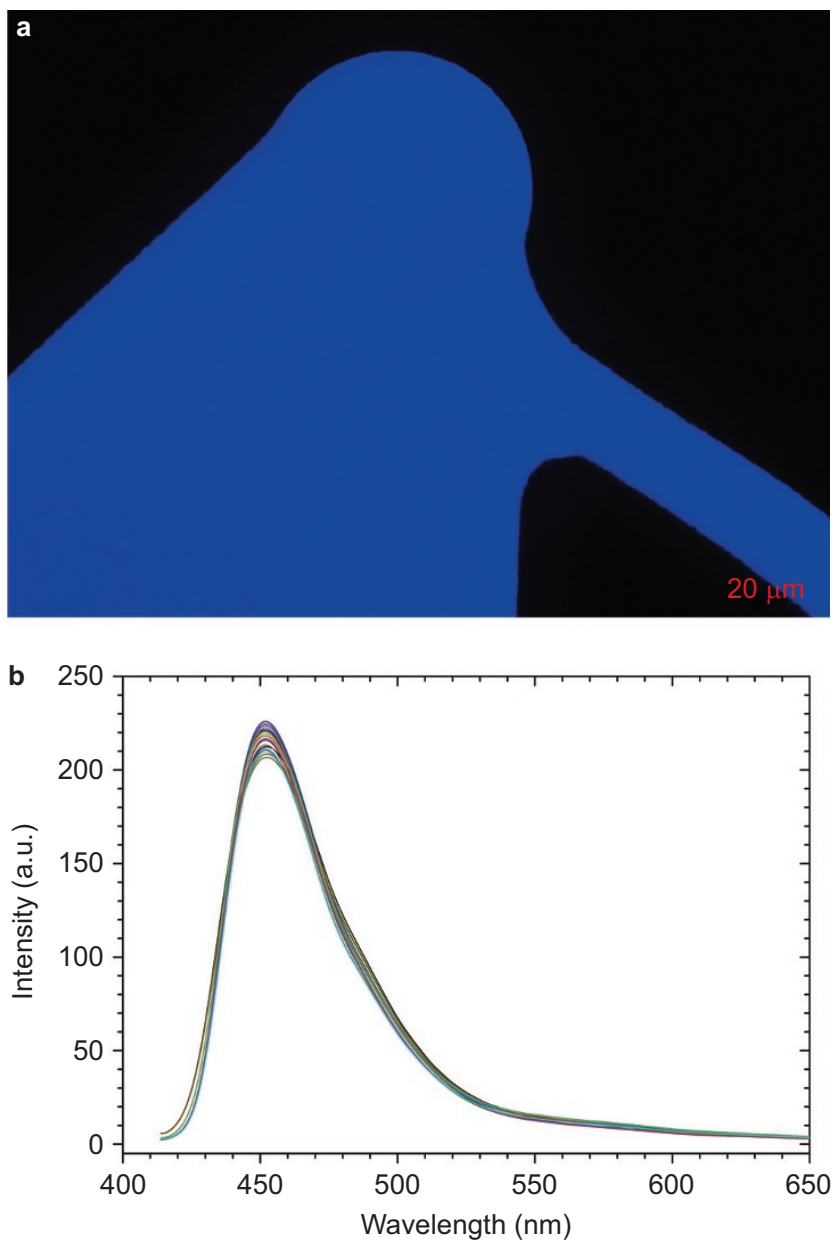

Figure 2 (a) Emission spectra recorded at 13 different points of the SSLE. (b) Image of the projection of the confocal images recorded every $5 \mu \mathrm{m}$ across the entire SSLE structure. SSLE, solid-state light emitter.

\section{Photostability studies of the SSLE}

The photostability study revealed that at low exposure times/radiation doses, fluctuations in the intensity signal occurred. The signal was stabilized after $15 \mathrm{~min}$ of total exposition time (Supplementary Fig. S2). With a total dose of $24.3 \mathrm{~J} \mathrm{~cm}^{-2}$, the decay in emission was only $4.1 \%$ of the initial value. These results corroborate the high photostability of Atto 390 and straightforwardly, of the SSLE presented here.

\section{Mapping studies}

The resulting map of maximum emitted intensities at $450 \mathrm{~nm}$ as a function of the coordinates of the external pumping light source spot is presented in the image capture shown in Figure 3a, in which the outline of the pear-shaped structure is indicated with a discontinuous line. As predicted by the numerical simulations, a symmetrical response was observed, with the maximum of coupled light obtained when exciting along the central longitudinal axis. The output intensity measured at $450 \mathrm{~nm}$ as a function of the Atto fluorophore concentration is shown in Figure 3b. The maximum intensity values increase with the fluorophore concentration. The linear trend observed confirms that autoabsorption phenomena did not occur at the concentrations used in this work.

The positioning tolerance of the external pumping light source over the SSLE is also of significant importance when considering integ- 
a
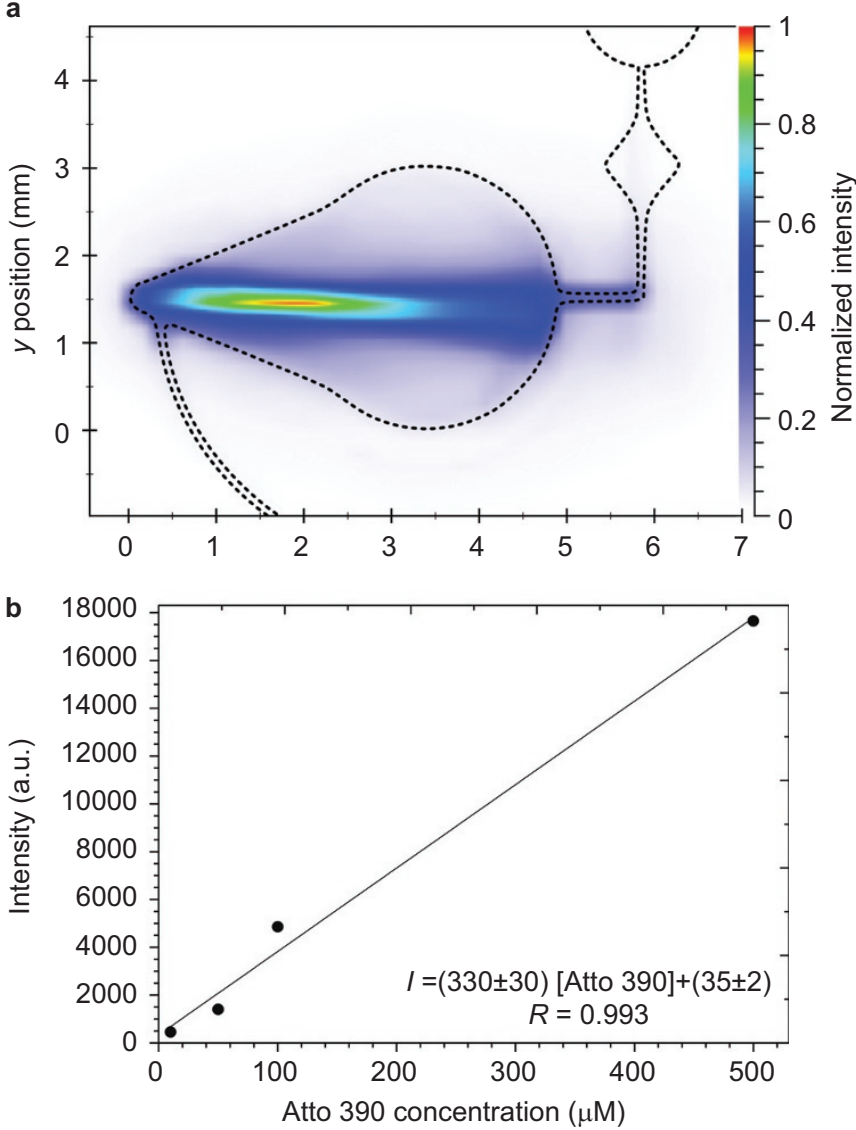

Figure 3 (a) Experimental emission intensity map study performed with the SSLE prepared with the $500 \mu \mathrm{M}$ Atto 390 solution. The outline of the pear-shaped structure is indicated by the discontinuous line for better visualization. (b) Maximum emitted intensity recorded at the optimal excitation point (highest emission) vs. Atto concentration. SSLE, solid-state light emitter.

ration of the PhLOC into a portable reading platform or into a future mass production system. To this effect, we have defined the longitudinal and transversal confidence positioning interval $\left(\eta_{\mathrm{L}}\right.$ and $\eta_{\mathrm{T}}$, respectively), the distance over which the external pumping light source spot can be moved while maintaining the output intensity at higher than $95 \%$ of the maximum SSLE emission intensity. The results are presented in Table 1 for all the fluorophore concentrations used. It can be concluded that whereas $\eta_{\mathrm{L}}$ (longitudinal to the SSLE axis) presents high tolerance against variations of the pumping excitation spot (positioning changes between 600 and $1125 \mu \mathrm{m}$ cause the emitted light intensity to vary $<5 \%$ ), the $\eta_{\mathrm{T}}$ tolerance is between half and one order of magnitude lower, which is consistent with both the mapping results presented above and the numerical results. Nevertheless, the $\eta_{\mathrm{T}}$ values $(150 \mu \mathrm{m})$ are large enough to be attained using massproduction technologies.

Table 1 Longitudinal and transversal confidence positioning intervals for each concentration

\begin{tabular}{lcc}
\hline Concentration $(\mu \mathrm{M})$ & $\begin{array}{c}\text { Longitudinal confidence } \\
\text { positioning interval } \eta_{\mathrm{L}}(\mu \mathrm{m})\end{array}$ & $\begin{array}{c}\text { Transversal confidence } \\
\text { positioning interval } \eta_{\mathrm{T}}(\mu \mathrm{m})\end{array}$ \\
\hline 10 & 1125 & 150 \\
50 & 600 & 150 \\
100 & 600 & 150 \\
500 & 1050 & 150 \\
\hline
\end{tabular}

\section{Analytical performance of the PhLOC}

Once the SSLE working principle was validated, the whole PhLOC was fabricated using the above described technology. Figure 4 presents an image of the final PhLOC under homogeneous wavelength excitation of $352 \mathrm{~nm}$. In this case, the MIR was filled with a dilution of $50 \mu \mathrm{M}$ fluorescein in PBS for better visualization. The extent of the homogeneity of the emission from the SSLE can again be observed. In addition, using the fabrication procedure described in this work, the SSLE is inherently self-aligned with both the interconnecting waveguide and the MIR, dramatically simplifying the implementation of the main components in any photonic system, namely, the light source, sensing region and detector (being in this case the SSLE, MIR and output fiber optics, respectively).

Absorbance measurements were performed using a non-biofunctionalized PhLOC. Quinoline yellow dye is a water-soluble acid dye that is extensively used as an additive in the food industry ${ }^{38}$ as well as a

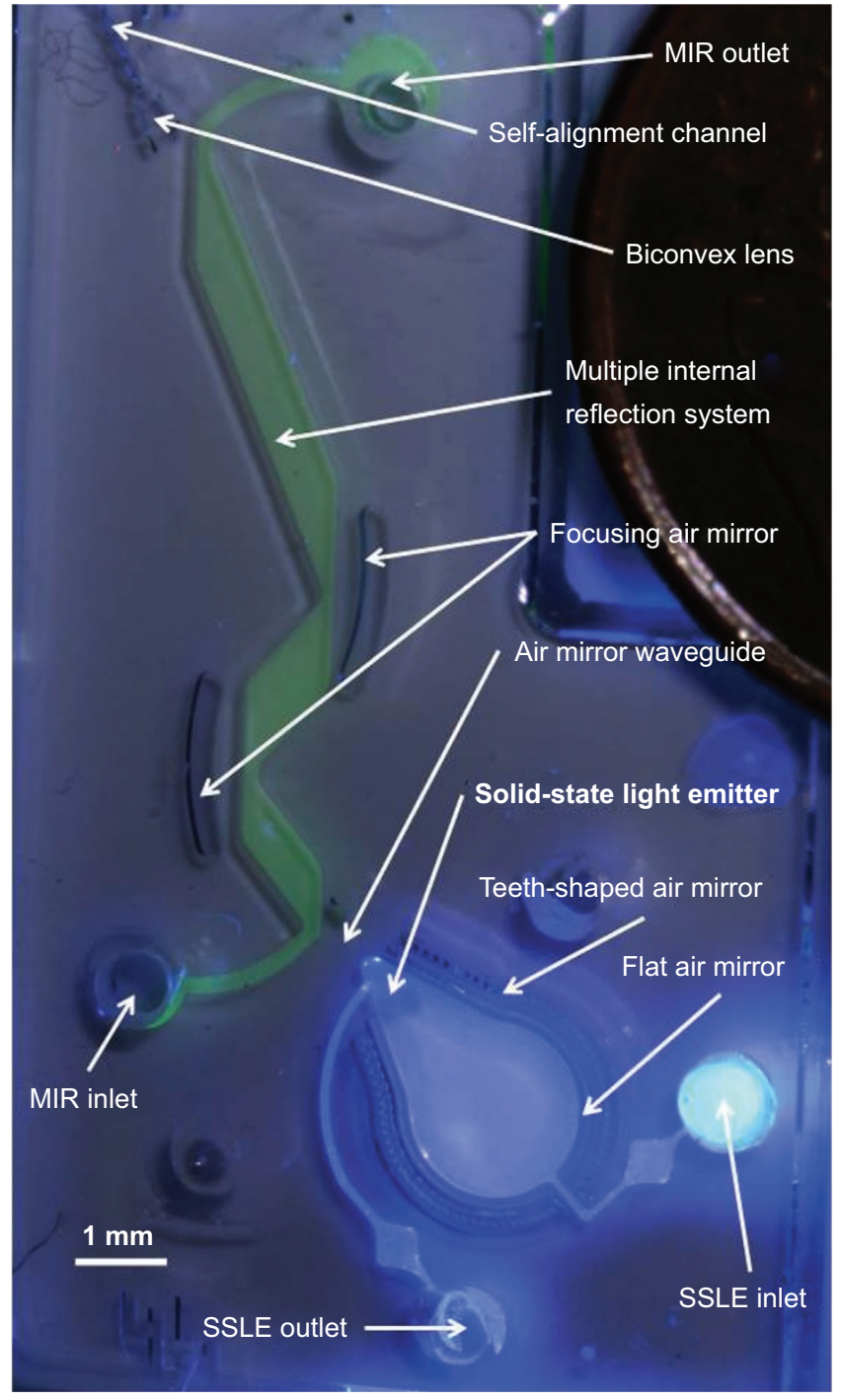

Figure 4 Fabricated PhLOC comprising the SSLE, air mirrors, MIR, biconvex lens and channel for fiber optics insertion. The MIR was filled with a PBS solution containing $50 \mu \mathrm{M}$ fluorescein only for visualization, and the entire system was placed under homogeneous 352-nm wavelength excitation. A 1-cent euro coin is also included for size reference. MIR, multiple internal reflection; PhLOC, photonic lab on a chip; SSLE, solid-state light emitter. 
coloring agent in general. ${ }^{39}$ This dye is also a potential biohazard, confirmed by genotoxicity tests using in vitro human lymphocytes. ${ }^{40}$ Therefore, the measurement of its concentration is not only important in the food industry but also in the environmental water analysis because of its deleterious effects, particularly for infants. ${ }^{39}$ Quinoline yellow dye has a maximum absorption wavelength at $\lambda_{\max }=440 \mathrm{~nm}$, which falls inside the Atto 390 emission spectra. The calibration curve obtained (Supplementary Fig. S3) using this PhLOC was in the linear range between 1 and $14 \mu \mathrm{M}$ with a LOD of $(0.60 \pm 0.01) \mu \mathrm{M}$. In comparison, Zhao et al. ${ }^{41}$ used a carbon nanotube-modified electrode to perform electrochemical characterization, obtaining a linear range between $2.74 \mu \mathrm{M}$ and $73.18 \mu \mathrm{M}$ and an LOD of $1.82 \mu \mathrm{M}$. Optical measurements were also provided by other authors, such as Sorouraddin and co-workers, ${ }^{42}$ who obtained a LOD of $3.5 \mu \mathrm{M}$. Only using a combination of chromatographic and spectrophotometric methods was a LOD of $0.4 \mu \mathrm{M}$ obtained, but this required a much more complex and expensive approach. Thus, the $\mathrm{PhLOC}$ reported here represents a dramatic improvement in the analysis of this target analyte compared with the state of the art. In addition, the hybrid monolithic integration of the SSLE allows for a simplification of its use and a reduction of the fabrication costs.

Finally, the performance of the biofunctionalized PhLOC for the detection of $\mathrm{H}_{2} \mathrm{O}_{2}$ was assessed. The working principle was based on the catalytic action of the HRP enzyme in the presence of an ABTS colorless charge transfer mediator. Upon the reduction of $\mathrm{H}_{2} \mathrm{O}_{2}$ to water, this mediator was concomitantly oxidized to the green-colored $\mathrm{ABTS}^{+\cdot}$ radical cation, which exhibited an intense absorption band from $390 \mathrm{~nm}$ to $445 \mathrm{~nm}$.

Three identical systems were functionalized and measured on three consecutive days, and the corresponding calibration curves are presented in Figure 5. The three systems presented a monotonic increase in the absorbance at a wavelength of $435 \mathrm{~nm}$ in solutions containing increasing concentrations of $\mathrm{H}_{2} \mathrm{O}_{2}$ ranging from $1.4 \mu \mathrm{M}$ to $8.2 \mu \mathrm{M}$, which is consistent with the behavior previously reported using a PhLOC with an external excitation light source. ${ }^{16}$ A linear fitting was performed in this range, and the estimated analytical parameters are presented in Table 2.

Good agreement is observed between the different measured PhLOCs, with a maximum variation of $10 \%$ in sensitivity and with absolute values similar to or better than those previously published for

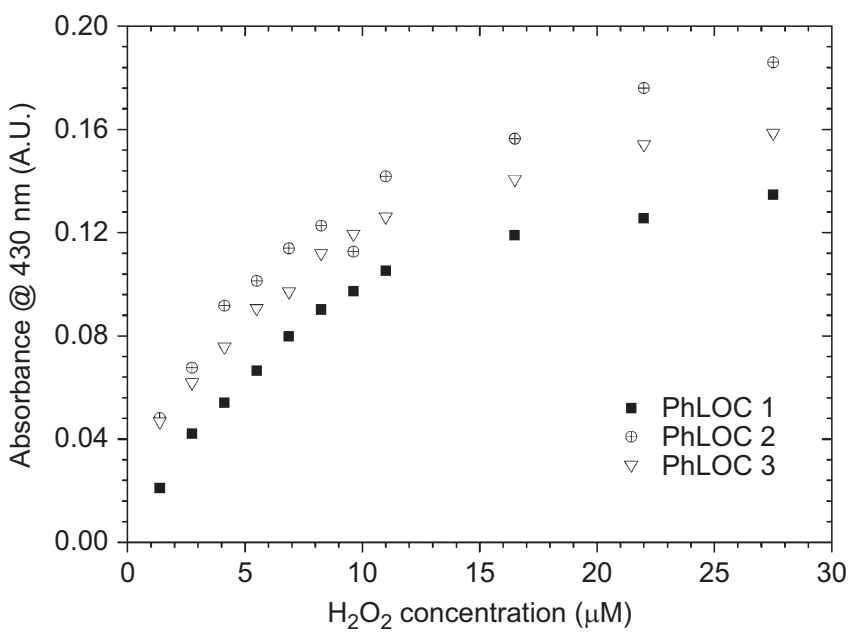

Figure 5 Absorbance as a function of $\mathrm{H}_{2} \mathrm{O}_{2}$ concentration measured at $435 \mathrm{~nm}$ using three different HRP-functionalized PhLOCs. PhLOC, photonic lab on a chip.
Table 2 Analytical data extracted from the $\mathrm{H}_{2} \mathrm{O}_{2}$ calibration curves performed with various PhLOCs

\begin{tabular}{lccc}
\hline & $\begin{array}{c}\text { Sensitivity } \\
\left(\text { A.U. } \mu \mathrm{M}^{-1}\right)\end{array}$ & LOD $(\mu \mathrm{M}) *$ & $R^{2}$ \\
\hline PhLOC \#1 & $0.0121 \pm 0.0005$ & $0.64 \pm 0.02$ & 0.992 \\
PhLOC \#2 & $0.0125 \pm 0.0006$ & $0.88 \pm 0.09$ & 0.989 \\
PhLOC \#3 & $0.0112 \pm 0.0005$ & $0.67 \pm 0.02$ & 0.991 \\
Mean & $0.0119 \pm 0.0007$ & $0.7 \pm 0.1$ & - \\
\hline
\end{tabular}

Abbreviations: LOD, limit of detection; PhLOC, photonic lab on a chip. * LOD calculated following the $3 \sigma$ IUPAC criteria using the linear concentration range from $1.4 \mu \mathrm{M}$ to $8.2 \mu \mathrm{M}$ for $\mathrm{H}_{2} \mathrm{O}_{2}$ detection. Absorbance recorded at $\lambda=435 \mathrm{~nm}$.

other optical biosensing approaches for $\mathrm{H}_{2} \mathrm{O}_{2} \cdot{ }^{16,43}$ These results confirm the feasibility of monolithically implementing a low-cost SSLE in a PhLOC without increasing the technological complexity while maintaining the performance of the other components of the system. In addition, even though this work was performed with Atto 390 fluorophore, any other molecule (or quantum dots) with photonic reemission could have been used instead, conferring to the proposed PhLOC an outstanding flexibility. Similarly, the use of HRP was selected to benchmark the PhLOC against a well-established enzymatic response. Nevertheless, other enzymes that are more clinically or biologically relevant could also be used in combination with this PhLOC approach.

\section{CONCLUSIONS}

We demonstrate the easy integration and successful performance of a PhLOC with a SSLE made of a fluorophore-doped hybrid xerogel material and a MIR as the main elements. The resulting photonic approach can be fabricated at low cost because the fabrication only requires one photolithographic step. Thus, the elements of such systems are inherently aligned. Coupling enhancement between the SSLE and MIR was achieved by defining air mirrors surrounding the SSLE to redirect emitted light toward the MIR. Confocal studies of the SSLE revealed homogeneous fluorophore dispersion. Using quinoline yellow as a colored target analyte, the feasibility of this approach was verified, obtaining a LOD of $(0.60 \pm 0.01) \mu \mathrm{M}$. Finally, the MIR was selectively biofunctionalized with HRP, and the PhLOC was benchmarked against hydrogen peroxide $\left(\mathrm{H}_{2} \mathrm{O}_{2}\right)$ as a target analyte, providing a LOD of $(0.7 \pm 0.1) \mu \mathrm{M}$ for $\mathrm{H}_{2} \mathrm{O}_{2}$. This detection strategy could be applied to other enzyme- or immunoassay-based analytical tools for the measurement of a wide range of target analytes and validates both the proposed $\mathrm{PhLOC}$ and its fabrication strategy.

\section{ACKNOWLEDGEMENTS}

The research leading to these results has received funding from the European Research Council under the European Community's Seventh Framework Programme (FP7/2007-2013)/ERC grant agreement no. 209243 and Spanish MINECO, project ref. TEC2010-17274. AG acknowledges the support received by CONACyT. SB gratefully acknowledges the financial support received by the Volkswagen Foundation.

1 Manz A, Graber N, Widmer HM. Miniaturized total chemical-analysis systems-a novel concept for chemical sensing. Sens Actuators B Chem 1990; 1: 244-248.

2 Witters D, Knez K, Ceyssens F, Puers R, Lammertyn J. Digital microfluidics-enabled single-molecule detection by printing and sealing single magnetic beads in femtoliter droplets. Lab Chip 2013; 13: 2047-2054.

3 Domachuk P, Tsioris K, Omenetto FG, Kaplan DL. Bio-microfluidics: biomaterials and biomimetic designs. Adv Mater 2010; 22: 249-260. 
4 Carlborg CF, Haraldsson T, Öberg K, Malkoch M, van der Wijngaart W. Beyond PDMS: off-stoichiometry thiol-ene (OSTE) based soft lithography for rapid prototyping of microfluidic devices. Lab Chip 2011; 11: 3136-3147.

5 Mark D, Haeberle S, Roth G, von Stetten F, Zengerle R. Microfluidic lab-on-a-chip platforms: requirements, characteristics and applications. Chem Soc Rev 2010; 39: 1153-1182.

6 Ramsey JM, Hadd AG, Jacobson SC. Applications of precise fluid control on microchips. In: Proceedings of International Conference on Solid-State Sensors and Actuators; 16-19 June 1997; Chicago, IL, USA. IEEE: New York, 1997, Vol. 1-2, pp919-921.

7 Wu MH, Huang SB, Lee GB. Microfluidic cell culture systems for drug research. Lab Chip 2010; 10: 939-956.

8 Schudel BR, Harmon B, Abhyankar VV, Pruitt BW, Negrete OA et al. Microfluidic platforms for RNA interference screening of virus-host interactions. Lab Chip 2013; 13: 811-817.

9 Menegatti E, Berardi D, Messina M, Ferrante I, Giachino 0 et al. Lab-on-a-chip: emerging analytical platforms for immune-mediated diseases. Autoimmunity Rev 2013; 12: 814-820.

10 Ma Z, Liu Q, Yang H, Runyan RB, Eisenberg CA et al. Laser patterning for the study of MSC cardiogenic differentiation at the single-cell level. Light Sci App/2013; 2: e68.

11 Xia YN, Whitesides GM. Soft lithography. Angew Chem Int Ed 1998; 37: 550-575.

12 Bodas D, Khan-Malek C. Hydrophilization and hydrophobic recovery of PDMS by oxygen plasma and chemical treatment-an SEM investigation. Sens Actuators $B$ Chem 2007; 123: 368-373.

13 Berdichevsky Y, Khandurina J, Guttman A, Lo YH. UV/ozone modification of poly(dimethylsiloxane) microfluidic channels. Sens Actuators B Chem 2004; 97 : 402-408.

14 Yu L, Li CM, Zhou Q, Luong JHT. Poly(vinyl alcohol) functionalized poly(dimethylsiloxane) solid surface for immunoassay. Bioconjugate Chem 2007; 18: 281-284.

15 Sandison ME, Cumming SA, Kolch W, Pitt AR. On-chip immunoprecipitation for protein purification. Lab Chip 2010; 10: 2805-2813.

16 Ibarlucea B, Fernández-Sánchez C, Demming S, Büttgenbach S, Llobera A. Selective functionalisation of PDMS-based photonic lab on a chip for biosensing. Analyst 2011; 136: 3496-3502.

17 Liu G, Khor SM, lyengar SG, Gooding JJ. Development of an electrochemical immunosensor for the detection of HbAlc in serum. Analyst 2012; 137: 829-832.

18 Liao JC, Mastali M, Li Y, Gau V, Suchard MA et al. Development of an advanced electrochemical DNA biosensor for bacterial pathogen detection. J Mol Diagn 2007; 9: 158-168.

19 Kimmel DW, LeBlanc G, Meschievitz ME, Cliffel DE. Electrochemical sensors and biosensors. Anal Chem 2012; 84: 685-707.

20 Hoshino K, Huang YY, Lane N, Huebschman M, Uhr JW et al. Microchip-based immunomagnetic detection of circulating tumor cells. Lab Chip 2011; 11: 3449-3457.

21 Balslev S, Jorgensen AM, Bilenberg B, Mogensen KB, Snakenborg D et al. Lab-on-achip with integrated optical transducers. Lab Chip 2006; 6: 213-217.

22 Lepage D, Jiménez A, Beauvais J, Dubowski JJ. Real-time detection of influenza A virus using semiconductor nanophotonics. Light Sci App/ 2013; 2: e62.

23 Llobera A, Demming S, Wilke R, Büttgenbach S. Multiple internal reflection poly(dimethylsiloxane) systems for optical sensing. Lab Chip 2007; 7: 1560-1566.

24 Llobera A, Wilke R, Büttgenbach $\mathrm{S}$. Enhancement of the response of poly (dimethylsiloxane) hollow prisms through air mirrors for absorbance-based sensing. Talanta 2008; 75: 473-479.

25 Llobera A, Wilke R, Büttgenbach S. Poly(dimethylsiloxane) hollow Abbe prism with microlenses for detection based on absorption and refractive index shift. Lab Chip 2004; 4: 24-27.

26 Liu R, Cai Y, Park JM, Ho KM, Shinar J et al. Organic light-emitting diode sensing platform: challenges and solutions. Adv Funct Mater 2011; 21: 4744-4753.
27 Vannahme C, Klinkhammer S, Lemmer U, Mappes T. Plastic lab-on-a-chip for fluorescence excitation with integrated organic semiconductor lasers. Opt Express 2011; 19: 8179-8186.

28 Lebeau B, Innocenzi P. Hybrid materials for optics and photonics. Chem Soc Rev 2011; 40: 886-906.

29 Zaggout FR. Entrapment of phenol red pH indicator into a sol-gel matrix. Mater Letters 2006; 60: 1026-1030.

30 Carregal-Romero E, Llobera A, Cadarso VJ, Darder M, Aranda P et al. One-step patterning of hybrid xerogel materials for the fabrication of disposable solid-state emitters. ACS Appl Mater Interfaces 2012; 4: 5029-5037.

31 Fernández-Sánchez C, Cadarso VJ, Darder M, Domínguez C, Llobera A. Patterning high-aspect-ratio sol-gel structures by micro transfer molding. Chem Mater 2008; 20: 2662-2668.

32 Llobera A, Cadarso VJ, Carregal-Romero E, Brugger J, Domínguez C et al. Fluorophoredoped xerogel antiresonant reflecting optical waveguides. Opt Express 2011; 19: 5026-5039.

33 Zhang XH, Que W, Jia CY, Ju JX, Liu WG. Fabrication of micro-lens arrays built in photosensitive hybrid films by UV-cured imprinting technique. J Sol-Gel Sci Technol 2011, 60: 71-80

34 Hench LL, West JK. The sol-gel process. Chem Rev 1990; 90: 33-72.

35 Vila-Planas J, Fernández-Rosas E, Ibarlucea B, Demming S, Nogués C et al. Cell analysis using a multiple internal reflection photonic lab-on-a-chip. Nat Protoc 2011; 6: 1642-1655.

36 Fernandez-Rosas E, Baldi A, Ibañez E, Barrios L, Novo S et al. Chemical functionalization of polysilicon microparticles for single-cell studies. Langmuir 2011; 27: 8302-8308.

37 Thomsen V, Schatzlein D, Mercuro D. Limits of detection in spectroscopy. Spectroscopy 2003; 18: 112-114

38 Capitan-Vallvey LF, Fernandez MD, deOrbe I, Vilchez JL, Avidad R. Simultaneous determination of the colorants sunset yellow FCF and quinolone yellow by solidphase spectrophotometry using partial least squares multivariate calibration. Analyst 1997; 122: 351-354.

39 Coleman M. Cancer Risk after Medical Treatment. New York: Oxford University Press; 1991.

40 Macioszek VK, Kononowicz AK. The evaluation of the genotoxicity of two commonly used food colors: quinoline yellow (E 104) and brilliant black BN (E 151). Cell Mol Biol Lett 2004; 9: 107-122.

41 Zhao J, Zhang Y, Wua K, Chen J, Zhou Y. Electrochemical sensor for hazardous food colourant quinoline yellow based on carbon nanotube-modified electrode. Food Chem 2011; 128: 569-572.

42 Gianotti V, Angioi S, Gosetti F, Marengo E, Gennaro MC. Chemometrically assisted development of IP-RP-HPLC and spectrophotometric methods for the identification and determination of synthetic dyes in commercial soft drinks. J Liquid Chromatogr Relat Technol 2005; 28: 923-937.

43 Ho WJ, Chen JS, Ker MD, Wu TK, Wu CY et al. Fabrication of a miniature CMOS-based optical biosensor. Biosens Bioelectron 2007; 22: 3008-3013.

(c) (i) $(-)$ This work is licensed under a Creative Commons Attributioncc) party material in this article are included in the article's Creative Commons license, unless indicated otherwise in the credit line; if the material is not included under the Creative Commons license, users will need to obtain permission from the license holder to reproduce the material. To view a copy of this license, visit http://creativecommons.org/licenses/ by-nc-nd/3.0/ 